\title{
On Solving Systems of Autonomous Ordinary Differential Equations by Reduction to a Variable of an Algebra
}

\author{
Alvaro Alvarez-Parrilla, ${ }^{1,2}$ Martín Eduardo Frias-Armenta, ${ }^{3}$ \\ Elifalet López-González, ${ }^{4}$ and Carlos Yee-Romero ${ }^{1}$ \\ ${ }^{1}$ Facultad de Ciencias, Universidad Autónoma de Baja California, Km. 103 Carretera Tijuana-Ensenada, \\ 22860 Ensenada, BC, Mexico \\ ${ }^{2}$ Grupo Alximia SA de CV, Departamento de Investigación, Ryerson 1268, Zona Centro, \\ 22800 Ensenada, BC, Mexico \\ ${ }^{3}$ Departamento de Matemáticas, Universidad de Sonora, 83000 Hermosillo, SON, Mexico \\ ${ }^{4}$ División Multidisciplinaria de la UACJ en Cuauhtémoc, Universidad Autónoma de Ciudad Juárez, \\ 32310 Ciudad Juárez, CHIH, Mexico
}

Correspondence should be addressed to Alvaro Alvarez-Parrilla, alvaro@uabc.edu.mx

Received 26 March 2012; Accepted 16 May 2012

Academic Editor: Mihai Putinar

Copyright (c) 2012 Alvaro Alvarez-Parrilla et al. This is an open access article distributed under the Creative Commons Attribution License, which permits unrestricted use, distribution, and reproduction in any medium, provided the original work is properly cited.

\begin{abstract}
A new technique for solving a certain class of systems of autonomous ordinary differential equations over $\mathbb{K}^{n}$ is introduced ( $\mathbb{K}$ being the real or complex field). The technique is based on two observations: (1), if $\mathbb{K}^{n}$ has the structure of certain normed, associative, commutative, and with a unit, algebras $\mathbb{A}$ over $\mathbb{K}$, then there is a scheme for reducing the system of differential equations to an autonomous ordinary differential equation on one variable of the algebra; (2) a technique, previously introduced for solving differential equations over $\mathbb{C}$, is shown to work on the class mentioned in the previous paragraph. In particular it is shown that the algebras in question include algebras linearly equivalent to the tensor product of matrix algebras with certain normal forms.
\end{abstract}

\section{Introduction}

Throughout this work, $\mathbb{K}$ will stand for a field, usually the real $\mathbb{R}$ or the complex field $\mathbb{C}$. Consider the autonomous ordinary differential equation

$$
\frac{d x}{d t}=f(x), \quad x \in \Omega \subset \mathbb{K}^{n}, t \in \mathbb{R},
$$

with $f: \Omega \subset \mathbb{K}^{n} \rightarrow \mathbb{K}^{n}$ having certain regularity conditions. 
In general the solution is not easy to obtain since this is usually a system of coupled differential equations. There is a vast literature regarding the solution of ordinary differential equations by different means and in particular by techniques utilizing generalized analytic functions, see for instance [1-14]. These include applications to the three-dimensional Stokes problem, solutions of planar elliptic vector fields with degeneracies, the Dirichlet problem, multidimensional stationary Schrödinger equation, among others $[3,5,6,12,13]$. In particular, the technique that we present is of interest for people working on vector fields with singularities. For instance, in order to gain insight into the behaviour of analytic vector fields, correct visualization of vector fields in the vicinity of their singular set is required, in the case of visualization of two-dimensional complex analytic vector fields with essential singularities the usual methods only provide partial results (see [15-17]), whilst the technique which we promote provides accurate and correct solutions $[18,19]$. These questions arise naturally in discrete and continuous dynamical systems (see [20-22]).

As a first step in obtaining a solution to (1.1), we notice that, if $\mathbb{K}^{n}$ can be given the structure of a certain algebra $\mathbb{A}$, it is possible to reduce this system to a single autonomous differential equation

$$
\frac{d \zeta}{d t}=\tilde{g}(\zeta), \quad \zeta \in \Omega^{\prime} \subset \mathbb{A}, t \in \mathbb{R}
$$

with $\widetilde{g}$ being a function $\mathbb{A}$-differentiable with respect to the variable $\zeta$ in the algebra $\mathbb{A}$.

Having done so, we proceed to show that it is possible to solve (1.2) by extending a geometric technique introduced in $[18,19]$ in the context of complex analytic vector fields related to Newton vector fields which are first studied by [23]. This technique is based upon the construction of two functions, which, are respectively, constant and linear on the trajectories which are the solutions of (1.2).

The paper is organized as follows. In Section 2 the algebras in question are introduced, in particular we introduce the notion of normed, associative, commutative, and with a unit, finite dimensional algebra $\mathbb{A}$ over $\mathbb{K}$, showing in Section 2.1 that these have a first fundamental representation into the algebra of $n \times n$ matrices over $\mathbb{K}, M(n, \mathbb{K})$. Furthermore in Section 2.2 normal algebras are defined and their corresponding tensor products are constructed. This is standard material which can be found in [24-26] but is presented here for completeness.

In Section 3 we give the definition of $\mathbb{A}$-differentiability, and proceed to show that if the family of matrices $\{J f(x): x \in \Omega\}$ is linearly equivalent to a subset of an algebra $\mathbb{B}$ in $M(n, \mathbb{K})$, that is, the image of the first fundamental representation of an algebra $\mathbb{A}$ with respect to the canonical basis of $\mathbb{K}^{n}$, that is, $\mathbb{B}=R(\mathbb{A})$, then in fact $f$ is $\mathbb{A}$-differentiable on $\Omega$. The problem of determining if a map $f: \Omega \subset \mathbb{K}^{n} \rightarrow \mathbb{K}^{n}$ is $\mathbb{A}$-differentiable for some algebra $\mathbb{A}$ is treated indirectly in [27], where the conditions that are given ensure the existence of an algebra $\mathbb{A}$ such that the set of relations are the generalized Cauchy-Riemann equations for $\mathbb{A},([27,28]$ show that these equations give a criterion for $\mathbb{A}$-differentiability). Furthermore [29] considers the case when $\mathbb{K}=\mathbb{C}$, where he proves that every analytic map $f$ (in the usual sense) which is $\mathbb{A}$-differentiable has an expansion in power series (see [29, pp. 646 and 653]).

In Section 4 we show that for normal algebras it is possible to express the function $f(x)$ in terms of a single variable $\zeta$ in the algebra, and hence there is a function $\widetilde{g}(\zeta)$ that represents $f(x)$. The analogy being the algebra of complex numbers, where $z=x+i y$ is the variable and the $\mathbb{A}$-differentiable functions being the analytic functions. We also show that there is a differentiable operator $\partial / \partial \zeta$ which has the property that $\partial \tilde{g} / \partial \zeta=\tilde{g}^{\prime}$, where $\tilde{g}^{\prime}$ is the Lorch derivative of $\tilde{g}$ (see [26]), hence providing a framework for the usual calculus of one variable. 

the form

In Section 5 we start by showing that in this context the differential equation (1.1) takes

$$
\frac{d \zeta}{d t}=\widetilde{g}(\zeta), \quad \zeta \in \Omega^{\prime} \backslash \mathcal{S} \subset \mathbb{A}, t \in \mathbb{R}
$$

with $\tilde{g}$ being a function $\mathbb{A}$-differentiable with respect to the variable $\zeta$ in the algebra $\mathbb{A}$, and $\mathcal{S}$ being a certain singular set, where the solutions are not defined.

We then proceed to show that the geometric technique, introduced in [18, 19], of finding two functions $h_{1}$ and $h_{2}$ which are constant and linear on the trajectories $\zeta(t)$ which are solutions of the differential equation, can be extended to the case of (1.3). We end the section and the paper with an example.

\section{Algebras}

We introduce $\mathbb{K}$-algebras (see, e.g., [24]).

Definition 2.1. A $\mathbb{K}$-algebra (or algebra over $\mathbb{K}$ ) is a finite dimensional $\mathbb{K}$-linear space $\mathbb{A}$ on which is defined a bilinear map $\mathbb{A} \times \mathbb{A} \rightarrow \mathbb{A}$ that is associative and commutative, and there is a unit element $e=e_{\mathbb{A}}$ in $\mathbb{A}$ that satisfies $e x=x e=x$ for all $x \in A$.

An element $a \in \mathbb{A}$ is called regular if there exist a unique element in $\mathbb{A}$ denoted by $a^{-1} \in \mathbb{A}$ called inverse of $a$ such that $a^{-1} a=a a^{-1}=e$. An element $a \in \mathbb{A}$ which is not regular is called singular. If $a, b \in \mathbb{A}$ and $b$ is regular, the quotient $a / b$ will mean $a b^{-1}$.

\subsection{Algebras and Their Fundamental Representations}

We define the first fundamental representation.

If $\boldsymbol{B}=\left\{\beta_{1}, \ldots, \beta_{n}\right\}$ is an ordered basis of an algebra $\mathbb{A}$, the product between the elements of $B$ is given by

$$
\beta_{i} \beta_{j}=\sum_{k=1}^{n} c_{i j k} \beta_{k}
$$

where $c_{i j k} \in \mathbb{K}$ for $i, j, k \in\{1,2, \ldots, n\}$ are called the structure constants of $\mathbb{A}$. The first fundamental representation of $\mathbb{A}$ associated to $B$ is the isomorphism $R: \mathbb{A} \rightarrow M(n, \mathbb{K})$ defined by

$$
R\left(x_{1} \beta_{1}+x_{2} \beta_{2}+\cdots+x_{n} \beta_{n}\right)=x_{1} R_{1}+x_{2} R_{2}+\cdots+x_{n} R_{n}
$$

where $R_{i}$ is the matrix whose entry $(j, k)$ is $c_{i j k}$ for $i=1,2, \ldots, n$. The commutativity and the associativity of $\mathbb{A}$ are equivalent to the identities

(1) $c_{i j k}=c_{j i k}$, for all $i, j, k \in\{1,2 \ldots, n\}$ and

(2) $\sum_{l} c_{i j l} c_{l k s}=\sum_{l} c_{j k l} c_{i l s}$, for all $i, j, k, s \in\{1,2 \ldots, n\}$,

respectively. 
Using $R$ we assign to $\mathbb{A}$ the norm induced from the operator norm in $M(n, \mathbb{K})$ (see [24]). In this way each algebra is a normed algebra, that is, there exists a norm $\|\cdot\|: \mathbb{A} \rightarrow \mathbb{R}$ satisfying $\|x y\| \leq\|x\|\|y\|$ for all $x, y \in \mathbb{A}$ and $\|e\|=1$.

Example 2.2. Let $\mathbb{A}$ be the linear space $\mathbb{R}^{3}$ with the product between the elements of the standard basis $\mathbb{B}=\left\{e_{1}, e_{2}, e_{3}\right\}$ given in the following equation:

\begin{tabular}{c|ccc} 
& $e_{1}$ & $e_{2}$ & $e_{3}$ \\
\hline$e_{1}$ & $e_{1}$ & $e_{2}$ & $e_{3}$ \\
$e_{2}$ & $e_{2}$ & $-2 e_{1}+2 e_{2}+e_{3}$ & $-e_{1}+e_{2}+e_{3}$ \\
$e_{3}$ & $e_{3}$ & $-e_{1}+e_{2}+e_{3}$ & $-e_{1}+2 e_{3}$
\end{tabular}

that extends to the product in $\mathbb{R}^{3}$ given by

$$
\begin{aligned}
(x, y, z)(u, v, w)= & \left(e_{1} x, e_{2} y, e_{3} z\right)\left(e_{1} u, e_{2} v, e_{3} w\right) \\
= & x u e_{1} e_{1}+x v e_{1} e_{2}+x w e_{1} e_{3}+y u e_{1} e_{2}+y v e_{2} e_{2} \\
& +y w e_{2} e_{3}+z u e_{1} e_{3}+z v e_{2} e_{3}+x w e_{3} e_{3} \\
= & (x u-2 y v-y w-z v-z w) e_{1} \\
& +(x v-y u-z y v-y w+z v) e_{2} \\
& +(z w+z u+y v+y w+z v+z w) e_{3} .
\end{aligned}
$$

The product between the elements of $\boldsymbol{B}$ define the structure constants $c_{i j k}$ for $i, j, k \in$ $\{1,2,3\}$. So, $\mathbb{R}^{3}$ with the product given is an algebra $\mathbb{A}$.

\subsection{Normal Algebras and Their Tensor Products}

Let $B_{1}, B_{2}, \ldots, B_{l}$ be matrices $B_{i} \in M\left(k_{i}, \mathbb{K}\right)$, each $B_{i}$ of one the following four types:

$$
(r),\left(\begin{array}{ccccc}
s & 0 & 0 & \cdots & 0 \\
1 & s & 0 & & 0 \\
0 & 1 & s & & 0 \\
\vdots & & & \ddots & \\
0 & 0 & 0 & \cdots & s
\end{array}\right),\left(\begin{array}{cc}
a & -b \\
b & a
\end{array}\right),\left(\begin{array}{ccccc}
C & 0 & 0 & \cdots & 0 \\
I_{2} & C & 0 & & 0 \\
0 & I_{2} & C & & 0 \\
\vdots & & & \ddots & \\
0 & 0 & 0 & \cdots & C
\end{array}\right)
$$

where

$$
C=\left(\begin{array}{cc}
u & -v \\
v & u
\end{array}\right), \quad I_{2}=\left(\begin{array}{ll}
1 & 0 \\
0 & 1
\end{array}\right)
$$


$r, s, a, b, u, v \in \mathbb{K}$ and $k_{1}+\cdots+k_{l}=n$. In this case we will say that the matrix $B$ given by

$$
B=\left(\begin{array}{cccc}
B_{1} & 0 & \cdots & 0 \\
0 & B_{2} & & 0 \\
\vdots & & \ddots & \\
0 & 0 & \cdots & B_{l}
\end{array}\right)
$$

is in its normal form. We will associate to $B$ an algebra of matrices of dimension $n$ over $\mathbb{K}$ which contains $B$ and use the following nomenclature: The first block will be called real simple block, the second real Jordan block, the third simple complex block, and the fourth complex Jordan block.

For $i=1,2, \ldots, l$, let $\sigma_{i}: M\left(k_{i}, \mathbb{K}\right) \rightarrow M(n, \mathbb{K})$ be the linear sections defined by substituting the matrix $M \in M\left(k_{i}, \mathbb{K}\right)$ in the block $B_{i}$ of the matrix $B$ and taking the other entries as zero. The real Jordan block may be written in the following way $B_{i}=a_{i} D_{i}+N_{i}$, where $D_{i}$ is the identity and $N_{i}$ is a nilpotent matrix of order $k_{i}$. The simple complex block may be written in the form $B_{i}=a_{i} D_{i}+b_{i} J_{i}$, where $D_{i}$ is a diagonal matrix and $J_{i}$ is a matrix with $J_{i}^{2}=-D_{i}$. The complex Jordan blocks may be written in the form $B_{i}=a_{i} D_{i}+b_{i} J_{i}+N_{i}$, where $D_{i}$ is the identity and $J_{i}$ is a matrix with $J_{i}^{2}=-D_{i}$ and $N_{i}$ is a nilpotent matrix of order $k_{i} / 2$.

We define the matrices $\left\{\beta_{i, j}: 1 \leq i \leq l, 1 \leq j \leq k_{i}\right\}$ whose entries are in the block $B_{i}$ as follows.

(i) If $B_{i}$ is a real simple block, $\beta_{i, 1}:=\sigma_{i}(1)$, in this case $k_{i}=1$.

(ii) If $B_{i}$ is a real Jordan block, $\beta_{i, 1}:=\sigma_{i}\left(D_{i}\right)$ and $\beta_{i, j}:=\sigma_{i}\left(N_{i}^{j-1}\right)$ for $j=2, \ldots, k_{i}$.

(iii) If $B_{i}$ is a simple complex block, $\beta_{i, 1}:=\sigma_{i}\left(D_{i}\right)$ and $\beta_{i, 2}:=\sigma_{i}\left(J_{i}\right)$.

(iv) If $B_{i}$ is a complex Jordan block, $\beta_{i, 1}:=\sigma_{i}\left(D_{i}\right), \beta_{i, 2}:=\sigma_{i}\left(J_{i}\right), \beta_{i, 2 j-1}:=\sigma_{i}\left(N_{i}^{2 j-1}\right)$, and $\beta_{i, 2 j}:=\beta_{i, 2} \sigma_{i}\left(N_{i}^{2 j-1}\right)$ for $j=2, \ldots, k_{i} / 2$.

Observe that the product of the matrices $\beta_{i_{1}, j_{1}}$ and $\beta_{i_{2}, j_{2}}$ is the zero matrix if $i_{1} \neq i_{2}$. The products of $\beta_{i, j_{1}}$ and $\beta_{i, j_{2}}$ for $i=1, \ldots, l$ are as follows.

(i) If $B_{i}$ is a real simple block, $\beta_{i, 1} \beta_{i, 1}=\beta_{i, 1}$.

(ii) If $B_{i}$ is a real Jordan block, then

$$
\begin{array}{c|cc} 
& \beta_{i, 1} & \beta_{i, j_{1}} \\
\hline \beta_{i, 1} & \beta_{i, 1} & \beta_{i, j_{1}} \\
\beta_{i, j_{2}} & \beta_{i, j_{1}} & \beta_{i, j_{1}+j_{2}}
\end{array}
$$

for $2 \leq j_{1}, j_{2} \leq k_{i}$, where $\beta_{i, j_{1}}=0$ when $j_{1} \geq k_{i}+1$.

(iii) If $B_{i}$ is a complex simple block, then

$$
\begin{array}{c|cc} 
& \beta_{i, 1} & \beta_{i, 2} \\
\hline \beta_{i, 1} & \beta_{i, 1} & \beta_{i, 2} \\
\beta_{i, 2} & \beta_{i, 2} & -\beta_{i, 1}
\end{array}
$$


(iv) If $B_{i}$ is a complex Jordan block, then

\begin{tabular}{c|cccc} 
& $\beta_{i, 1}$ & $\beta_{i, 2}$ & $\beta_{i, 2 j_{1}-1}$ & $\beta_{i, 2 j_{1}}$ \\
\hline$\beta_{i, 1}$ & $\beta_{i, 1}$ & $\beta_{i, 2}$ & $\beta_{i, 2 j_{1}-1}$ & $\beta_{i, 2 j_{1}}$ \\
$\beta_{i, 2}$ & $\beta_{i, 2}$ & $-\beta_{i, 1}$ & $\beta_{i, 2 j_{1}}$ & $-\beta_{i, 2 j_{1}-1}$ \\
$\beta_{i, 2 j_{2}-1}$ & $\beta_{i, 2 j_{2}-1}$ & $\beta_{i, 2 j_{2}}$ & $\beta_{i, 2\left(j_{1}+j_{2}-1\right)-1}$ & $\beta_{i, 2\left(j_{1}+j_{2}-1\right)}$ \\
$\beta_{i, 2 j_{2}}$ & $\beta_{i, 2 j_{2}}$ & $-\beta_{i, 2 j_{2}-1}$ & $\beta_{i, 2\left(j_{1}+j_{2}-1\right)}$ & $-\beta_{i, 2\left(j_{1}+j_{2}-1\right)-1}$
\end{tabular},

for $2 \leq j_{1}, j_{2} \leq k_{i} / 2$, where $\beta_{i, j}=0$ when $j \geq 2 k_{i}+1$.

The commutativity of the elements in the set

$$
\mathbb{B}=\left\{\beta_{1,1}, \ldots, \beta_{1, k_{1}}, \ldots, \beta_{l, 1}, \ldots, \beta_{l, k_{l}}\right\},
$$

with respect to the matrix product, follows from the well-known result: For a Jordan canonical form $D+N$ the diagonal matrix $D$ commutes with the nilpotent matrix $N$.

Moreover, the $\mathbb{K}$-linear space spanned by $\mathbb{B}$ is an $\mathbb{K}$-algebra, as is claimed in the following proposition.

Proposition 2.3. The set $B:=\left\{\beta_{1,1}, \ldots, \beta_{1, k_{1}}, \ldots, \beta_{l, 1}, \ldots, \beta_{l, k_{l}}\right\}$ is a base for an $n$-dimensional linear space which is an algebra $\mathbb{A}$ with respect to the matrix product, and its first fundamental representation $R$ with respect to $B$ is the identity isomorphism.

Proof. Let $R$ be the first fundamental representation of $\mathbb{A}$ associated to $\mathbb{B}$. As the algebra $\mathbb{A}$ is generated by $\beta_{i, 1}, \beta_{i, 2}$ (considered only when this exists, i.e., $k_{i} \geq 2$ ), and $\beta_{i, 3}$ (considered only in the case when $B_{i}$ is a Jordan complex block), in order to prove that $R$ is the identity, we only need to prove that $R\left(\beta_{i, j}\right)=\beta_{i, j}$, but for these three cases the equality is trivial. So, $R$ is the identity isomorphism.

Definition 2.4. Given a matrix $B \in M(n, \mathbb{K})$ in its normal form, one will call the algebra in $M(n, \mathbb{K})$, as constructed above, an $\mathbb{K}$-normal algebra (containing $B$ ).

Definition 2.5. Two matrix algebras $\mathbb{A}_{1}$ and $\mathbb{A}_{2}$ in $M(n, \mathbb{K})$ are linearly equivalent if there exists an invertible matrix $B \in M(n, \mathbb{K})$ such that $\mathbb{A}_{1}=\left\{B A B^{-1}: A \in \mathbb{A}_{2}\right\}$.

For a proof of the following result, see [30].

Proposition 2.6. Let $\mathbb{A}$ and $\mathbb{B}$ be algebras. There exists a product in $\mathbb{A} \otimes \mathbb{B}$ satisfying

$$
\left(a_{1} \otimes b_{1}\right)\left(a_{2} \otimes b_{2}\right)=a_{1} a_{2} \otimes b_{1} b_{2}
$$

where $a_{1} a_{2}$ and $b_{1} b_{2}$ denote the products in $\mathbb{A}$ and $\mathbb{B}$, respectively. The product is associative and $e_{\mathbb{A}} \otimes e_{\mathbb{B}}=e_{\mathbb{A} \otimes \mathbb{B}}$.

Therefore, the finite tensor product of algebras is an algebra.

Definition 2.7. The complexification of an $\mathbb{R}$-algebra $\mathbb{A}$ is the $\mathbb{C}$-algebra $\mathbb{A} \otimes \mathbb{C}$. One calls an algebra which is the complexification of an $\mathbb{R}$-normal algebra a $\mathbb{C}$-normal algebra. 
As usual, if the context is clear, one will drop the $\mathbb{C}$ from the name and refer to the $\mathbb{C}$-normal algebra just as a normal algebra. The following proposition and its corollary follow from a straightforward calculation.

Proposition 2.8. Let $\mathbb{A}$ and $\mathbb{B}$ be $p$-and q-dimensional matrix algebras in $M(n, \mathbb{K})$ and $M(m, \mathbb{K})$, respectively, and $P \in M(n, \mathbb{K})$ and $Q \in M(m, \mathbb{K})$ be invertible matrices. Then, one has

$$
P \mathbb{A} P^{-1} \otimes Q \mathbb{B} Q^{-1}=[P \otimes Q][\mathbb{A} \otimes \mathbb{B}][P \otimes Q]^{-1}
$$

Corollary 2.9. The tensor product of matrix algebras linearly equivalent to normal algebras is algebras which are linearly equivalent to the tensor product of normal algebras.

So, by Corollary 2.9 the algebras linearly equivalent to the tensor product of normal algebras are closed under the tensor product.

The following result shows that the first fundamental representation, with respect to an appropriate base, of a tensor product of normal algebras is the inclusion of the algebra in the corresponding matrix space.

Proposition 2.10. Let $\mathbb{A}$ and $\mathbb{B}$ be $p$ - and $q$-dimensional $\mathbb{K}$-algebras, and $R_{1}: \mathbb{A} \rightarrow M(p, \mathbb{K})$ and $R_{2}: \mathbb{B} \rightarrow M(q, \mathbb{K})$ be first fundamental representations associated to the basis $\mathcal{A}=\left\{\alpha_{1}, \ldots, \alpha_{p}\right\}$ and $\mathbb{B}=\left\{\beta_{1}, \ldots, \beta_{q}\right\}$, respectively. Then, $R: \mathbb{A} \otimes \mathbb{B} \rightarrow M(p q, \mathbb{K})$ defined by $R=R_{1} \otimes R_{2}$ is the first fundamental representation of $\mathbb{A} \otimes \mathbb{B}$ associated to the base $\left\{\alpha_{i} \otimes \beta_{j}: 1 \leq j \leq p, 1 \leq j \leq q\right\}$.

Proof. Let $\left\{\alpha_{1}, \ldots, \alpha_{p}\right\}$ be a base of $\mathbb{A}$ and let $\left\{\beta_{1}, \ldots, \beta_{q}\right\}$ be a base of $\mathbb{B}$. We use the notations $C_{i}$ and $G_{j}$ for the matrices $R_{1}\left(\alpha_{i}\right)$ and $R_{2}\left(\beta_{j}\right)$, respectively, for $1 \leq i \leq p, 1 \leq j \leq q$. The set

$$
\left\{\alpha_{i} \otimes \beta_{j}: 1 \leq i \leq p, 1 \leq j \leq q\right\}
$$

is a base for $\mathbb{A} \otimes \mathbb{B}$ (see [30]). In order to find the structure constants of $\mathbb{A} \otimes \mathbb{B}$ we take the products

$$
\begin{aligned}
{\left[\alpha_{i} \otimes \beta_{l}\right]\left[\alpha_{j} \otimes \beta_{s}\right] } & =\alpha_{i} \alpha_{j} \otimes \beta_{l} \beta_{s} \\
& =\left(\sum_{k=1}^{p} c_{i j k} \alpha_{k}\right) \otimes\left(\sum_{t=1}^{q} d_{l s t} \beta_{t}\right) \\
& =\sum_{k=1}^{p} \sum_{t=1}^{q} c_{i j k} d_{l s t}\left(\alpha_{k} \otimes \beta_{t}\right),
\end{aligned}
$$

from which we obtain a first fundamental representation $R$ of $A \otimes B$, where $R\left(\alpha_{i} \otimes \beta_{l}\right)$ is the matrix $H_{i l}$ whose entry $(k l, j s)$ is given by $h_{i l, k t, j s}:=c_{i j k} d_{l s t}$, where $c_{i j k}$ and $d_{l s t}$ are the entries $(k, j)$ and $(t, s)$ of $C_{i}$ and $G_{l}$ for $1 \leq i \leq p$ and $1 \leq l \leq q$, respectively. 
On the other hand we have that $R_{1}\left(\alpha_{i}\right) \otimes R_{2}\left(\beta_{l}\right)=C_{i} \otimes G_{l}$. The tensor product of $C_{i}$ and $G_{l}$ is given by

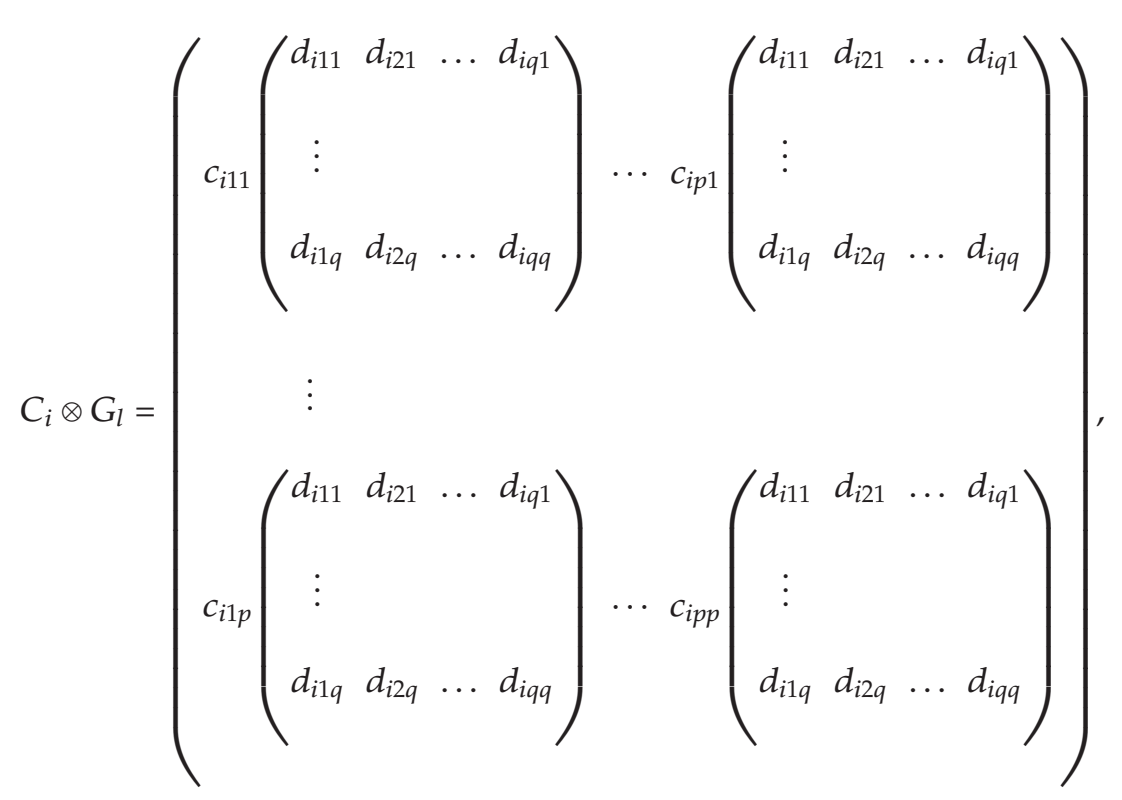

from which we see that in the position $(k t, j s)$ the element $c_{i j k} d_{l s t}$ appears. Thus, we have the equalities of matrices $H_{i l}=C_{i} \otimes G_{l}$, for $1 \leq i, l \leq n$. Therefore, $R=R_{1} \otimes R_{2}$.

Corollary 2.11. If $\mathbb{A}$ is a matrix algebra in $M(n, \mathbb{K})$ which is a tensor product of $\mathbb{K}$-normal algebras, then there exists a base $\mathbb{B}$ of $\mathbb{A}$ in which the corresponding first fundamental representation $R: \mathbb{A} \rightarrow$ $M(n, \mathbb{K})$ is the identity isomorphism, that is, $R(x)=x$ for all $x \in \mathbb{A}$.

Proof. We have that $\mathbb{A}=\mathbb{B}_{1} \otimes \cdots \otimes \mathbb{B}_{m}$, where $\mathbb{B}_{i}$ is a $\mathbb{K}$-normal algebra for every $i \in$ $\{1, \ldots, m\}$. Obviously, for every $i \in\{1, \ldots, m\}$ we can consider a base for $\mathbb{B}_{i}$ as that given in Proposition 2.3, and taking the corresponding tensor products of these basis we obtain a base $\mathbb{B}$ for $\mathbb{A}$. By Propositions 2.3 and 2.10 we have that the first fundamental representation of $\mathbb{A}$ associated to $B$ is the identity.

\section{Differentiability on Algebras}

In a paper published in 1893, Sheffers laid a foundation for a theory of analytic functions on algebras, see [27, 29] and references therein.

Differentiability on algebras is a stronger concept than the usual differentiability over $\mathbb{K}^{n}$. If $f: U \subset \mathbb{K}^{n} \rightarrow \mathbb{K}^{n}$ is a differentiable map in the open set $U$, we denote by $J f(x)$ its Jacobian matrix at the point $x$, in the standard base of $\mathbb{K}^{n}$. We also use the notation $J_{\mathcal{B}} f(x)$ for the Jacobian matrix at the point $x$ of $f$ with respect to a base $B$. 
The following definition was introduced in [26].

Definition 3.1. Let $\mathbb{A}$ be an algebra and let $f: \Omega \subset \mathbb{A} \rightarrow \mathbb{A}$ be a map defined in the open set $\Omega$. One says that $f$ is $\mathbb{A}$-differentiable at $x_{0} \in \mathbb{A}$ if there exists an element $f^{\prime}\left(x_{0}\right) \in \mathbb{A}$, which one calls the $\mathbb{A}$-derivative of $f$ at $x_{0}$, satisfying

$$
\lim _{h \rightarrow 0} \frac{\left\|f\left(x_{0}+h\right)-f\left(x_{0}\right)-f^{\prime}\left(x_{0}\right) h\right\|}{\|h\|}=0,
$$

where $f^{\prime}\left(x_{0}\right) h$ denotes the product in $\mathbb{A}$ of $f^{\prime}\left(x_{0}\right)$ with $h$. If $f$ is $\mathbb{A}$-differentiable at all the points of $\Omega$, one says that $f$ is $\mathbb{A}$-differentiable on $\Omega$ and one calls the map $f^{\prime}$ assigning $f^{\prime}(x)$ to the point $x \in \Omega$ the $\mathbb{A}$-derivative of $f$, or Lorch derivative of $f$.

It follows (see, e.g., [26-29]) that a map $f: \Omega \subset \mathbb{A} \rightarrow \mathbb{A}$ is $\mathbb{A}$-differentiable at $x_{0}$ if and only if $J_{\mathcal{B}} f\left(x_{0}\right) \in R(\mathbb{A})$ and is continuous as a function of $x_{0}$, where $\mathbb{B}:=\left\{e_{1}, \ldots, e_{n}\right\}$ is a base of $\mathbb{A}$ and $R: \mathbb{A} \rightarrow M(n, \mathbb{K})$ is the first fundamental representation of the algebra $\mathbb{A}$ associated to $B$.

In fact, in this case, $J_{B} f(x)$ is the image of $f^{\prime}(x)$ under the map $R$, that is, if $f$ is $\mathbb{A}$ differentiable, then

$$
J_{\mathbb{B}} f(x)=\sum_{i=1}^{n} u_{i}(x) R_{i}
$$

where $R_{i}=R\left(e_{i}\right)$ and $u_{i}: \Omega \rightarrow \mathbb{K}$ for $i=1,2, \ldots, n$.

If $\Omega^{\prime}:=R(\Omega), \mathbb{B}:=R(\mathbb{A})$, and $g: \Omega^{\prime} \subset \mathbb{B} \rightarrow \mathbb{B}$ is defined by

$$
g(y)=R \circ f \circ R^{-1}(y)
$$

then $g$ is $\mathbb{B}$-differentiable and its differential at $y$ is given by $g^{\prime}(y)=\sum_{i=1}^{n} u_{i}\left(R^{-1}(y)\right) R_{i}$, thus, the relation between the Jacobian matrix of $f$ and the $\mathbb{B}$-differential of $g$ is $J_{\mathbb{B}} f(x)=g^{\prime}(R(x)$ ).

The matrix equation (3.2) is equivalent to the $n^{2}$ equations

$$
\frac{\partial f_{i}}{\partial x_{j}}=\sum_{l=1}^{n} u_{l} c_{l j i}
$$

for all $i, j \in\{1,2, \ldots, n\}$. Using (3.4) and the associativity of the algebra, we can obtain

$$
\sum_{i=1}^{n} c_{i k s} \frac{\partial f_{i}}{\partial x_{j}}=\sum_{i=1}^{n} c_{i j s} \frac{\partial f_{i}}{\partial x_{k}}
$$

appearing in $[29$, p. 646].

Remark 3.2. It should be noted that in the context of algebras equations (3.5) play the same role as the Cauchy-Riemann equations in the case of one complex variable and thus serve as a criterion for analyticity, see [27, 28, 31-33]. 
Suppose we consider a basis $\mathcal{A}=\left\{\alpha_{1}, \ldots, \alpha_{n}\right\}$ of $\mathbb{A}$, where $\alpha_{i}=\sum_{j=1}^{n} s_{j i} e_{j}$ for $i=1, \ldots, n$, $s_{j i} \in \mathbb{K}$, it can be proved that $J_{\mathscr{A}} f=S^{-1}(J f) S$, where $S=\left(s_{i j}\right)$. If we denote by $S_{i}$ the image of $\alpha_{i}$ under the first fundamental representation of $\mathbb{A}$ associated to $A$, we have for $i=1, \ldots, n$, that $S_{i}=S^{-1}\left(\sum_{j=1}^{n} S_{j i} R_{j}\right) S$.

Remark 3.3. For the differentiation of algebras the usual properties of differentiation of functions from $\mathbb{R}^{n}$ to $\mathbb{R}^{m}$ remain true. Furthermore, the usual rules of differentiation of functions of one variable are satisfied in the case of algebras, therefore polynomial functions, rational functions, and those expressed by means of convergent power series as the exponential, trigonometric, and other usual functions are differentiable in algebras.

The following theorem gives conditions that ensure the existence of an algebra $\mathbb{A}$ in which $f$ is $\mathbb{A}$-differentiable.

Theorem 3.4. Let $f: \Omega \subset \mathbb{K}^{n} \rightarrow \mathbb{K}^{n}$ be a $C^{1}$ map defined in the open set $\Omega$. $f$ is $\mathbb{A}$-differentiable for an algebra $\mathbb{A}$ if and only if the set of matrices

$$
\{J f(x): x \in \Omega\}
$$

is a subset of an algebra $\mathbb{B}$ which is linearly equivalent to an algebra $\mathbb{T}$ which is a finite tensor product of normal algebras in $M(n, \mathbb{K})$. Moreover, $\mathbb{A}$ is a $\mathbb{K}$-linear space $\mathbb{K}^{n}$ and has a base $\mathbb{B}$ such that the image of the first fundamental representation of $\mathbb{A}$ associated to $B$ is $R(\mathbb{A})=\mathbb{T}$.

Proof. Let $\mathcal{A}=\left\{\alpha_{1}, \ldots, \alpha_{n}\right\}$ be a base for $\mathbb{T}$ as given in Corollary 2.11. Then $\mathbb{B}=$ $\left\{B \alpha_{1} B^{-1}, \ldots, B \alpha_{n} B^{-1}\right\}$ is a base for $\mathbb{B}$, where $B$ is a matrix such that $\mathbb{B}=B \mathbb{T} B^{-1}$. Because $J f(x) \in \mathbb{B}$ for every $x \in \Omega$, we have $J f(x)=\sum_{i=1}^{n} u_{i}(x) \beta_{i}$, where $u_{i}: \Omega \rightarrow \mathbb{K}$ are functions and $\beta_{i}=B \alpha_{i} B^{-1}$.

Now consider the base $\mathcal{G}=\left\{\gamma_{1} \ldots, \gamma_{n}\right\}$ of $\mathbb{K}^{n}$ defined by $\gamma_{i}=\sum_{j=1}^{n} b_{j i} e_{j}$, where $B=\left(b_{i j}\right)$ and $\left\{e_{1}, \ldots, e_{n}\right\}$ is the standard base of $\mathbb{K}^{n}$. Then, we have that $J_{\mathcal{G}} f=B^{-1}(J f) B$. Thus,

$$
\begin{aligned}
J_{\mathcal{G}} f(x) & =B^{-1}\left(\sum_{i=1}^{n} u_{i}(x) \beta_{i}\right) B=B^{-1}\left(\sum_{i=1}^{n} u_{i}(x)\left(B \alpha_{1} B^{-1}\right)\right) B \\
& =\sum_{i=1}^{n} u_{i}(x) \alpha_{i}
\end{aligned}
$$

in other words $J_{\mathcal{G}} f(x) \in \mathbb{T}$, which means that if we define a product between the elements of $\mathcal{G}$ using the structure constants of the products of the elements of $\mathcal{A}$, we have that $\mathbb{K}^{n}$ is an algebra $\mathbb{A}$ such that its first fundamental representation associated to $\mathcal{G}$ is that given by $R\left(\gamma_{i}\right)=\alpha_{i}$ for $i=1, \ldots, n$. Therefore $f$ is $\mathbb{A}$-differentiable.

\section{Reduction to a Variable in the Algebra}

Sometimes $f=\left(f_{1}, \ldots, f_{n}\right): \Omega \subset \mathbb{K}^{n} \rightarrow \mathbb{K}^{n}$ can be expressed as a function of a variable $\zeta=\sum_{i=1}^{n} x_{i} e_{i}$ of an algebra $\mathbb{A}$ whose image under the first fundamental representation is the tensor product of normal algebras (as in the previous sections). In this section we show some necessary conditions for this to be true and also allow for the expression of $f$ in terms of $\zeta$. 
Proposition 4.1. With the same hypothesis as Theorem 3.4, there exists a basis $\boldsymbol{\Phi}=\left\{\delta_{1}, \ldots, \delta_{n}\right\}$ of invertible elements of the algebra $\mathbb{A}$ such that the following diagram commutes

$$
\begin{array}{lll}
\Omega \subset \mathbb{K}^{n} & \stackrel{f}{\longrightarrow} & \mathbb{K}^{n} \\
A \downarrow & & \downarrow A \\
\Omega^{\prime} \subset \mathbb{A} & \stackrel{\tilde{g}}{\longrightarrow} & \mathbb{A},
\end{array}
$$

where $A$ is the matrix associated to the change of basis $\varepsilon \rightarrow \Phi$, where $\varepsilon=\left\{e_{1}, \ldots, e_{n}\right\}$ is the canonical basis of $\mathbb{K}^{n}$.

Proof. By a change of basis one has the following commutative diagram

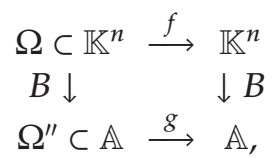

where $B$ is the matrix associated to the change of basis from $\varepsilon$ to $B$, which was used in the previous theorem, and $g=B \circ f \circ B^{-1}$. Now consider a base $\Phi=\left\{\delta_{1}, \ldots, \delta_{n}\right\}$ of regular elements of $\mathbb{A}$, where without loss of generality $\delta_{1}=e$. Then if $A$ is the matrix associated to the change of basis from $\mathcal{\varepsilon}$ to $\Phi$, let $\tilde{g}=A \circ f \circ A^{-1}$. Note that the domain of $\tilde{g}$ is $\Omega^{\prime}=A(\Omega)$.

Theorem 4.2. Let $f: \Omega \subset \mathbb{K}^{n} \rightarrow \mathbb{K}^{n}$ be a $\mathbb{A}$-differentiable map over an algebra $\mathbb{A}$ with first fundamental representation $R(\mathbb{A})$, then $\tilde{g}=A \circ f \circ A^{-1}$ can be expressed in a variable $\zeta=\sum_{j=1}^{n} y_{j} \delta_{j}$, for some regular basis $\Phi=\left\{\delta_{1}, \ldots, \delta_{n}\right\}$. Moreover there is a partial differential operator $\partial / \partial \zeta$ such that $\tilde{g}^{\prime}(\zeta)=(\partial \tilde{g} / \partial \zeta)(\zeta)$.

Proof. By Proposition 4.1 there is a basis $\Phi=\left\{\delta_{1}, \ldots, \delta_{n}\right\}$ of regular elements of the algebra $\mathbb{A}$, with $\delta_{1}=e$, without loss of generality we may assume that $\left\|\delta_{j}\right\|=1$. Introducing the variable $\zeta$ in the base $\Phi$ as $\zeta=\sum_{i=1}^{n} y_{i} \delta_{i}=\sum_{i=1}^{n} x_{i} e_{i}$, we then have $f\left(x_{1}, \ldots, x_{n}\right)=\widetilde{g}(\zeta)$, with $\tilde{g}=A \circ f \circ A^{-1}$, where $A$ is the matrix associated to the change of basis $\varepsilon$ to $\boldsymbol{\Phi}$.

Denote by $\overline{\zeta_{k}}$ the $k$-conjugate of $\zeta$ related to $\Phi$, which is defined for $k=2, \ldots, n$ by

$$
\begin{aligned}
\overline{\zeta_{k}} & =y_{1} \delta_{1}-y_{k} \delta_{k}+\sum_{\substack{j=2 \\
j \neq k}}^{n} y_{j} \delta_{j} \\
& =-y_{k} \delta_{k}+\sum_{\substack{j=1 \\
j \neq k}}^{n} y_{j} \delta_{j}=\zeta-2 y_{k} \delta_{k} .
\end{aligned}
$$

Then we have

$$
y_{k} e=\frac{\zeta-\overline{\zeta_{k}}}{2} \delta_{k}^{-1}, \quad \text { for } k=2, \ldots, n
$$


and since

$$
y_{1} \delta_{1}=\zeta-\sum_{j=2}^{n} y_{j} \delta_{j}=\zeta-\sum_{j=2}^{n} \frac{\zeta-\overline{\zeta_{j}}}{2}
$$

then,

$$
y_{1} e=\frac{1}{2}\left((3-n) \zeta+\sum_{j=2}^{n} \overline{\zeta_{j}}\right) \delta_{1}^{-1}
$$

Furthermore one can define the differential operators

$$
\begin{gathered}
\frac{\partial}{\partial \zeta}=\frac{1}{n} \sum_{j=1}^{n} \delta_{j}^{-1} \frac{\partial}{\partial y_{j}}, \\
\frac{\partial}{\partial \overline{\zeta_{k}}}=\frac{1}{2}\left\{\delta_{1}^{-1} \frac{\partial}{\partial y_{1}}-\delta_{k}^{-1} \frac{\partial}{\partial y_{k}}\right\}, \quad \text { for } k=2, \ldots, n,
\end{gathered}
$$

which satisfy

$$
\begin{gathered}
\frac{\partial \zeta}{\partial \zeta}=1 \quad \frac{\partial \overline{\zeta_{k}}}{\partial \zeta}=\frac{n-2}{n} \quad \text { for } k=2, \ldots, n, \\
\frac{\partial \overline{\zeta_{k}}}{\partial \overline{\zeta_{k}}}=1 \quad \frac{\partial \zeta}{\partial \overline{\zeta_{k}}}=\frac{\partial \overline{\zeta_{j}}}{\partial \overline{\zeta_{k}}}=0 \quad \text { for } k=2, \ldots, n, k \neq j .
\end{gathered}
$$

If $f$ is $\mathbb{A}$-differentiable, $\tilde{g}$ is also $\mathbb{A}$-differentiable, hence

$$
\tilde{g}^{\prime}(\zeta)=\left.\frac{d \widetilde{g}}{d t}\left(\zeta+t \delta_{k}\right)\right|_{t=0}=\left.\frac{d \tilde{g}}{d t}\left(\zeta+t \delta_{j}\right)\right|_{t=0}, \quad \forall j, k,
$$

and since

$$
\left.\frac{d \tilde{g}}{d t}\left(\zeta+t \delta_{k}\right)\right|_{t=0}=\delta_{k}^{-1} \frac{\partial \tilde{g}}{\partial y_{k}}(\zeta)
$$

then for $k=2, \ldots, n$

$$
\frac{\partial \tilde{g}}{\partial \overline{\zeta_{k}}}(\zeta)=\frac{1}{2} \sum_{i=1}^{n}\left\{\delta_{1}^{-1} \frac{\partial g_{i}}{\partial y_{1}}(\zeta)-\delta_{k}^{-1} \frac{\partial g_{i}}{\partial y_{k}}(\zeta)\right\} \delta_{i}=0
$$

so the right hand expression of $\tilde{g}(\zeta)=\sum_{i=1}^{n} g_{i}(\zeta) \delta_{i}$ does not depend on the conjugated variables. In this way we obtain an expression $\tilde{g}(\zeta)$ depending only on $\zeta$, and not on $\overline{\zeta_{k}}$ for $k=2, \ldots, n$. 
Moreover, by (4.7), (4.10), and (4.11) one has that

$$
\tilde{g}^{\prime}(\zeta)=\frac{\partial \tilde{g}}{\partial y_{1}}(\zeta)=\frac{\partial \tilde{g}}{\partial \zeta}(\zeta)
$$

In the following example we show how we can reduce the variables of a map by substituting for variables in an algebra.

Example 4.3. Let $f\left(x_{1}, x_{2}, x_{3}\right)=\left(x_{1}^{2}, 2 x_{1} x_{2}, x_{2}^{2}+2 x_{1} x_{3}\right)$. Then

$$
J f=\left(\begin{array}{ccc}
2 x_{1} & 0 & 0 \\
2 x_{2} & 2 x_{1} & 0 \\
2 x_{3} & 2 x_{2} & 2 x_{1}
\end{array}\right)
$$

the $J f$ is in normal form, hence $f$ is $\mathbb{A}$-differentiable. If

$$
N=\left(\begin{array}{lll}
0 & 0 & 0 \\
1 & 0 & 0 \\
0 & 1 & 0
\end{array}\right)
$$

and $I$ is the identity, the multiplication in the associated algebra $R(\mathbb{A})$ is given by multiplication of matrices $I, N$, and $N^{2}$ representing, respectively, $R\left(e_{1}\right), R\left(e_{2}\right)$, and $R\left(e_{3}\right)$, where $\mathcal{E}=\left\{e_{1}, e_{2}, e_{3}\right\}$ is the canonical basis of $\mathbb{R}^{3}$. It is easy to see that $e_{2}$ and $e_{3}$ do not have inverse in the algebra $\mathbb{A}$. Consider the basis of regular elements

$$
\Phi=\left\{\delta_{1}=e_{1}, \delta_{2}=e_{1}+e_{2}, \delta_{3}=e_{1}+e_{3}\right\}
$$

we see that the matrix associated to the change of basis from $\mathcal{\varepsilon}$ to $\Phi$ is

$$
A=\left(\begin{array}{ccc}
1 & -1 & -1 \\
0 & 1 & 0 \\
0 & 0 & 1
\end{array}\right),
$$

so $f$ is transformed to $\tilde{g}=A \circ f \circ A^{-1}$,

$$
\begin{aligned}
\tilde{g}\left(y_{1} \delta_{1}+y_{2} \delta_{2}+y_{3} \delta_{3}\right)= & \left(y_{1}^{2}-2 y_{2}^{2}-2 y_{2} y_{3}-y_{3}^{2}\right) \delta_{1} \\
& +2 y_{2}\left(y_{1}+y_{2}+y_{3}\right) \delta_{2} \\
& +\left(y_{2}^{2}+2 y_{3}\left(y_{1}+y_{2}+y_{3}\right)\right) \delta_{3}
\end{aligned}
$$

Thus

$$
\zeta=y_{1} \delta_{1}+y_{2} \delta_{2}+y_{3} \delta_{3}
$$


is the variable in the algebra $\mathbb{A}$. Now consider the conjugates

$$
\overline{\zeta_{2}}=y_{1} \delta_{1}-y_{2} \delta_{2}+y_{3} \delta_{3}, \quad \overline{\zeta_{3}}=y_{1} \delta_{1}+y_{2} \delta_{2}-y_{3} \delta_{3}
$$

Then

$$
\begin{aligned}
& y_{1} e=\frac{1}{2}\left(\overline{\zeta_{2}}+\overline{\zeta_{3}}\right) \delta_{1}^{-1}, \\
& y_{2} e=\frac{1}{2}\left(\zeta-\overline{\zeta_{2}}\right) \delta_{2}^{-1}, \\
& y_{3} e=\frac{1}{2}\left(\zeta-\overline{\zeta_{3}}\right) \delta_{3}^{-1},
\end{aligned}
$$

so by substituting this in $\tilde{g}$ and simplifying we obtain

$$
\tilde{g}(\zeta)=\zeta^{2}
$$

the function in the variable of the algebra.

\section{Solving Systems of Ordinary Differential Equations by Reduction to One Variable on an Algebra}

By following $[18,19]$, we obtain, as direct corollaries of Theorem 3.4, Proposition 4.1, and Theorem 4.2, the following results.

Corollary 5.1. Let

$$
\begin{gathered}
f: \Omega \subset \mathbb{R}^{n} \longrightarrow \mathbb{R}^{n}, \\
\tilde{g}: \Omega^{\prime} \subset \mathbb{A} \longrightarrow \mathbb{A},
\end{gathered}
$$

be as in Theorem 4.2. Then there exists $\Phi: \Omega^{\prime} \subset \mathbb{A} \rightarrow \mathbb{A}$ which is $\mathbb{A}$-differentiable on $\Omega^{\prime} \backslash \mathcal{S}$, where $\mathcal{S}$ is a singular set, such that

$$
\begin{gathered}
\tilde{g}(\zeta)=-\frac{\Phi(\zeta)}{\Phi^{\prime}(\zeta)}, \\
f(x)=-[J \phi(x)]^{-1} \phi(x),
\end{gathered}
$$

with $\phi(x)=(\Phi \circ A)(x)$.

Proof. We need to show that there exists $\Phi: \Omega^{\prime} \subset \mathbb{A} \rightarrow \mathbb{A}$ such that

$$
\tilde{g}(\zeta)=-\frac{\Phi(\zeta)}{\Phi^{\prime}(\zeta)}
$$


Noticing that

$$
\log (\Phi(\zeta))^{\prime}=\frac{\Phi^{\prime}(\zeta)}{\Phi(\zeta)}=-\frac{1}{\tilde{g}(\zeta)}
$$

and by Remark 3.3, it follows that

$$
\Phi(\zeta)=\exp \left[-\int^{\zeta} \frac{d z}{\widetilde{g}(z)}\right]
$$

for $\zeta \in \Omega^{\prime} \backslash \mathcal{S}$, where

$$
\mathcal{S}=\left\{\zeta \in \Omega^{\prime}: \tilde{g}(\zeta) \notin \mathbb{A}^{*}\right\},
$$

with $\mathbb{A}^{*}$ being the regular elements of $\mathbb{A}$.

Remark 5.2. Note that $\mathcal{S}$ can be many things. For instance if $\tilde{g}(\zeta)=c \zeta$ with $c \notin \mathbb{A}^{*}$, then $\mathcal{S}=\mathbb{A}$. On the other hand, if $\tilde{g}(\zeta)$ is the polynomial function $g(\zeta)=c_{n} \zeta^{n}+\cdots+c_{1} \zeta+c_{0}$ with $c_{n}$ singular, then $\tilde{g}(\zeta)$ may be regular for all $\zeta \in \mathbb{A}$, (see [26, p. 418]), in which case $\mathcal{S}=\emptyset$.

Remark 5.3. As a special case one notices that if $\mathbb{A}=\mathbb{C}$ then $1 / \tilde{g}$ will be a complex analytic function on $\Omega^{\prime} \backslash \mathcal{S}$ hence $\mathcal{S}$ consists of isolated points (the isolated singularities of $1 / \tilde{g}$ ). This has been studied in $[18,19]$.

Remark 5.4. In case that $\mathcal{S} \neq \Omega^{\prime}$, then $\Omega^{\prime} \backslash S$ is an open dense set in $\Omega^{\prime}$. This is true since the set $\mathbb{A}^{*}$ is an open dense set in $\mathbb{A}$ hence for a continuous $\tilde{g}$ one has that $\widetilde{g}^{-1}\left(\mathbb{A}^{*}\right)$ is an open dense subset of $\Omega^{\prime}$.

Remark 5.5. By the previous remark, in case that $\mathcal{S} \neq \Omega^{\prime}$, then $\Phi(\zeta)$ exists for $\zeta \in \Omega^{\prime} \backslash \mathcal{S}$ even though it might be a multivalued function defined on each component of $\zeta \in \Omega^{\prime} \backslash \mathcal{S}$.

Remark 5.6. Further characterization and properties of $\mathcal{S}$ will be studied elsewhere. In what follows we assume that $\mathcal{S} \neq \Omega^{\prime}$.

Let $x=\left(x_{1}, x_{2}, \ldots, x_{n}\right) \in \mathbb{R}^{n} \backslash\{0\}$, then consider the projection onto $S^{n-1}$

$$
\prod(x)=\frac{x}{\|x\|} \in S^{n-1} .
$$

Corollary 5.7. Let $f: \Omega \subset \mathbb{R}^{n} \rightarrow \mathbb{R}^{n}$ be $\mathbb{A}$-differentiable in an algebra with first fundamental representation $R(\mathbb{A})$. Then the solutions to the differential equation,

$$
\frac{d x}{d t}=f(x)
$$


correspond to the level curves of the function

$$
\widetilde{h_{1}}(x)=\Pi(\phi(x))
$$

moreover the real-valued function,

$$
\widetilde{h_{2}}(x)=\log \|\phi(x)\|
$$

is a linear function of t along the solutions $x(t)$ of (5.8).

Proof. By Theorem 3.4, Proposition 4.1, and Theorem 4.2, the solutions $x(t)$ of (5.8) are in correspondence with the solutions $\zeta(t)$ of

$$
\frac{d \zeta}{d t}=\tilde{g}(\zeta)
$$

On the other hand, Corollary 5.1 shows that

$$
\Phi(\zeta(t))=\Phi\left(\zeta\left(t_{0}\right)\right) \exp \left[-t-t_{0}\right]
$$

the result follows immediately by applying $\Pi(\cdot)$ and $\log \|\cdot\|$ to (5.12).

Corollary 5.8. In particular, to visualize the trajectory that passes through the point $x_{0} \in \Omega \backslash A^{-1}(\mathcal{S})$ at time $t_{0} \in \mathbb{R}$, one needs only to plot the level curve $\left\{x \in \Omega: \widetilde{h_{1}}(x)=\widetilde{h_{1}}\left(x_{0}\right)\right\}$. Moreover one can find explicitly the point $x\left(t_{1}\right)$, for $t_{1} \in \mathbb{R}$, as the intersection of the level curve $\widetilde{h}_{1}(x)=\widetilde{h}_{1}\left(x_{0}\right)$ and the hypersurface $\tilde{h}_{2}(x)=\tilde{h}_{2}\left(x_{0}\right)-t_{1}+t_{0}$.

Example 5.9. Consider the function

$$
f(x, y)=(u(x, y), v(x, y))
$$

where

$$
\begin{gathered}
u(x, y)=\frac{1}{6}\left(2+6 x+\frac{-22+20 x+7 y}{4+5 x^{2}+8 x(-1+y)+y(-4+5 y)}\right. \\
\left.+\frac{5(2+4 x+5 y)}{4+5 x^{2}+8 x(1+y)+y(4+5 y)}\right) \\
v(x, y)=\frac{1}{6}\left(-4+6 y-\frac{5(-4+5 x+4 y)}{4+5 x^{2}+8 x(-1+y)+y(-4+5 y)}\right. \\
\left.+\frac{4-7 x-20 y}{4+5 x^{2}+8 x(1+y)+y(4+5 y)}\right)
\end{gathered}
$$


International Journal of Mathematics and Mathematical Sciences

Let

$$
A=\left(\begin{array}{ll}
1 & 2 \\
2 & 1
\end{array}\right)
$$

and notice that

$$
A \cdot J f\left(A^{-1}(x, y)\right) \cdot A^{-1}=\left(\begin{array}{cc}
a(x, y) & b(x, y) \\
-b(x, y) & a(x, y)
\end{array}\right)
$$

with

$$
\begin{aligned}
a(x, y)= & 1-\frac{2}{x^{2}+(-2+y)^{2}}+\frac{x(-6+4 x+3 y)}{\left(x^{2}+(-2+y)^{2}\right)^{2}} \\
& +\frac{x(4 x-3(2+y))}{\left(x^{2}+(2+y)^{2}\right)^{2}}-\frac{2}{x^{2}+(2+y)^{2}} \\
b(x, y)= & \frac{4 y}{\left(x^{4}+\left(-4+y^{2}\right)^{2}+2 x^{2}\left(4+y^{2}\right)\right)^{2}} \\
& \times\left[48-96 x-24 x^{2}-16 x^{3}-9 x^{4}+2 x^{5}+2(-3+2 x)\left(4+x^{2}\right) y^{2}+(3+2 x) y^{4}\right] .
\end{aligned}
$$

Hence $A \cdot J f\left(A^{-1}(x, y)\right) \cdot A^{-1}$ belongs to the normal algebra $\mathbb{C}$, so the previous results are available.

Thus

$$
\tilde{g}(x, y)=\left(A \circ f \circ A^{-1}\right)(x, y)=\left(u_{1}(x, y), v_{1}(x, y)\right),
$$

with

$$
\begin{aligned}
& u_{1}(x, y)=-1+x+\frac{6-4 x-3 y}{2\left(x^{2}+(-2+y)^{2}\right)}+\frac{6-4 x+3 y}{2\left(x^{2}+(2+y)^{2}\right)} \\
& v_{1}(x, y)=\frac{x\left(-12+12 x+x^{3}\right) y+2\left(-2+x^{2}\right) y^{3}+y^{5}}{x^{4}+\left(-4+y^{2}\right)^{2}+2 x^{2}\left(4+y^{2}\right)}
\end{aligned}
$$

is just a (complex) analytic function, since

$$
J \tilde{g}(x, y)=A \cdot J f\left(A^{-1}(x, y)\right) \cdot A^{-1} \in R(\mathbb{C}),
$$


so by letting $z=x+i y$, we have

$$
\tilde{g}(z)=u_{1}\left(\frac{z+\bar{z}}{2}, \frac{z-\bar{z}}{2 i}\right)+i v_{1}\left(\frac{z+\bar{z}}{2}, \frac{z-\bar{z}}{2 i}\right)=\frac{2-z^{2}+z^{3}}{4+z^{2}}
$$

By Corollary 5.1 one has

$$
\Phi(z)=\exp \left[-\int^{z} \frac{d \zeta}{\widetilde{g}(\zeta)}\right]
$$

so that

$$
\Phi(z)=\left(\frac{z-1-i}{z-1+i}\right)^{i}(z+1)^{-1}=\frac{e^{2 \arctan (1-z)}}{z+1} .
$$

Note that in $\mathbb{R}^{2}$ one has $\Pi(x, y)=\exp [i \operatorname{Arg}(x, y)]$ hence the level curves of $\Pi(\cdot)$ are in correspondence with the level curves of $\operatorname{Arg}(\cdot)$. So

$$
\begin{aligned}
h(z)=\operatorname{Arg}(\Phi(z))= & -\arctan \left(\frac{y}{1+x}\right) \\
& +\frac{1}{2} \log \left(1-\frac{4 y}{2+(-2+x) x+y(2+y)}\right)
\end{aligned}
$$

is a constant of motion $h$ associated to $\widetilde{g}(z)$ and the one associated to $f(x, y)$ is

$$
\begin{aligned}
\tilde{h}(x, y)=(h \circ A)(x, y)= & -\arctan \left(\frac{2 x+y}{1+x+2 y}\right) \\
& +\frac{1}{2} \log \left(1-\frac{4(2 x+y)}{2+5 x^{2}+y(-2+5 y)+x(2+8 y)}\right) .
\end{aligned}
$$

So by Corollary 5.8 the trajectories associated to

$$
\left(x^{\prime}(t), y^{\prime}(t)\right)=f(x(t), y(t))
$$

are the level curves of $\tilde{h}(x, y)$. These are presented in Figure 1. 
Table 1: Coordinates of the intersection of the level curves $\tilde{h}(x, y)=-1.68195$ and $\tilde{h}_{2}(x, y)=2.26841-t$ for $t=0,1,2,3,4$.

\begin{tabular}{lc}
\hline Time $(t)$ & $x_{t}=(x(t), y(t))$ \\
\hline 0 & $(0.863,-0.783)$ \\
1 & $(0.4808,-0.0710)$ \\
2 & $(0.2002,0.2548)$ \\
3 & $(0.0678,0.4654)$ \\
4 & $(91.1109,-52.307)$ \\
\hline
\end{tabular}

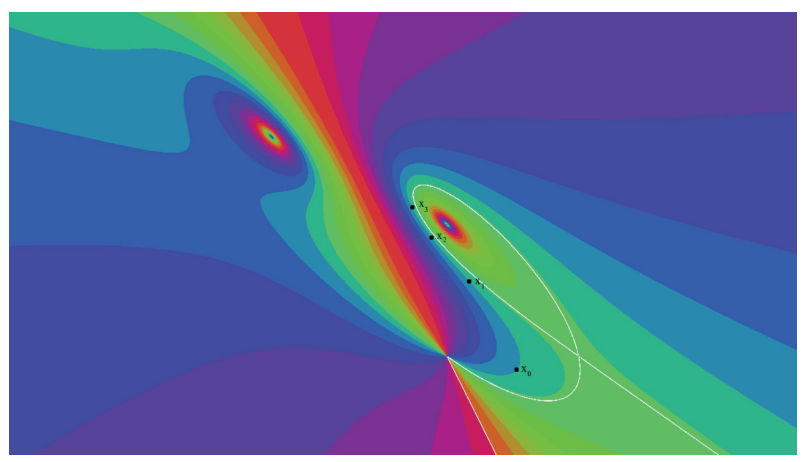

Figure 1: Visualization of the trajectories of (5.26). In this case we have plotted level bands of $\tilde{h}(x, y)$, so that the trajectories are in fact the boundaries between the colored bands. We have also plotted, in white, the trajectory that passes through a singular point corresponding to a pole of the (complex) analytic function $\tilde{g}(z)$. To exemplify the parametrization, we have plotted in black the points corresponding to $t=0,1,2,3$ (see Table 1). be

In order to parametrize the solution we need to calculate $\tilde{h}_{2}(x, y)$ which turns out to

$$
\begin{aligned}
\tilde{h}_{2}(x, y)= & -\arctan \left(\frac{-1+x+2 y}{1-2 x-y}\right) \\
& +\arctan \left(\frac{1-x-2 y}{1+2 x+y}\right) \\
& -\frac{1}{2} \log \left((2 x+y)^{2}+(1+x+2 y)^{2}\right) .
\end{aligned}
$$

According to Corollary 5.8 we proceeded to calculate the intersection the level curves $\tilde{h}(x, y)=-1.68195$ and $\tilde{h}_{2}(x, y)=2.26841-t$ for $t=0,1,2,3,4$. The results are shown in Table 1 and as black dots in Figure 1.

\section{Acknowledgments}

The first author wishes to thank Jesús Muciño-Raymundo for introducing him to the study of complex vector fields and for many interesting, insightful, and productive discussions. This work partially funded by UABC Grant no. 0196, and by CONACYT Grant CB-2010/150532. 


\section{References}

[1] L. Bers, Theory of Pseudo-Analytic Functions, Institute for Mathematics and Mechanics, New York University, New York, NY, USA, 1953.

[2] L. Bers, "An outline of the theory of pseudoanalytic functions," Bulletin of the American Mathematical Society, vol. 62, pp. 291-331, 1956.

[3] M. Düz and K. Koca, "A Dirichlet problem for generalized analytic functions," Selçuk Journal of Applied Mathematics, vol. 7, no. 1, pp. 9-15, 2006.

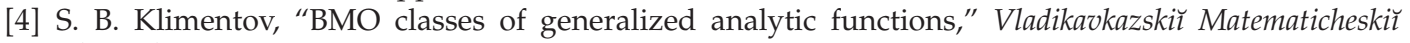
Zhurnal, vol. 8, no. 1, pp. 27-39, 2006.

[5] V. Kravchenko, "On the relationship between $p$-analytic functions and Schrödinger equation," Zeitschrift für Analysis und ihre Anwendungen, vol. 24, no. 3, pp. 487-496, 2005.

[6] A. Meziani, "Elliptic planar vector fields with degeneracies," Transactions of the American Mathematical Society, vol. 357, no. 10, pp. 4225-4248, 2005.

[7] Le Thu Hoai and W. Tutschke, "Associated spaces defined by ordinary differential equations," Zeitschrift für Analysis und ihre Anwendungen, vol. 25, no. 3, pp. 385-392, 2006.

[8] W. Tutschke, "Interactions between partial differential equations and generalized analytic functions," Cubo, vol. 6, no. 1, pp. 281-292, 2004.

[9] W. Tutschke, "Generalized analytic functions in higher dimensions," Georgian Mathematical Journal, vol. 14, no. 3, pp. 581-595, 2007.

[10] I. N. Vekua, Generalized analytic functions, Pergamon Press, Addison-Wesley, London, UK, 1962.

[11] Z.-1. Xu, "Mixed elastico-plasticity problems with partially unknown boundaries," Acta Mathematicae Applicatae Sinica (English Series), vol. 23, no. 4, pp. 629-636, 2007.

[12] M. Zabarankin and P. Krokhmal, "Generalized analytic functions in 3D Stokes flows," The Quarterly Journal of Mechanics and Applied Mathematics, vol. 60, no. 2, pp. 99-123, 2007.

[13] M. Zabarankin, "The framework of $k$-harmonically analytic functions for three-dimensional Stokes flow problems. I," SIAM Journal on Applied Mathematics, vol. 69, no. 3, pp. 845-880, 2008.

[14] B. Ziemian, "Generalized analytic functions with applications to singular ordinary and partial differential equations," Dissertationes Mathematicae (Rozprawy Matematyczne), vol. 354, 100 pages, 1996.

[15] K. Hockett and S. Ramamurti, "Dynamics near the essential singularity of a class of entire vector fields," Transactions of the American Mathematical Society, vol. 345, no. 2, pp. 693-703, 1994.

[16] T. Newton and T. Lofaro, "On using flows to visualize functions of a complex variable," Mathematics Magazine, vol. 69, no. 1, pp. 28-34, 1996.

[17] A. Garijo, A. Gasull, and X. Jarque, "Local and global phase portrait of equation $\dot{z}=\mathrm{f}(z)$," Discrete and Continuous Dynamical Systems A, vol. 17, no. 2, pp. 309-329, 2007.

[18] A. Alvarez-Parrilla, A. Gómez-Arciga, and A. Riesgo-Tirado, "Newton vector fields on the plane and on the torus," Complex Variables and Elliptic Equations, vol. 54, no. 5, pp. 440-461, 2009.

[19] A. Alvarez-Parrilla, J. Mucino-Raymundo, S. Solorza-Calderon, and C. Yee-Romero, Complex Analytic Vector Fields: Geometry, Dynamics and Visualization, 2009.

[20] H. E. Benzinger, "Julia sets and differential equations," Proceedings of the American Mathematical Society, vol. 117 , no. 4, pp. 939-946, 1993.

[21] F. von Haeseler and H. Kriete, "The relaxed Newton's method for rational functions," Random and Computational Dynamics, vol. 3, no. 1-2, pp. 71-92, 1995.

[22] F. von Haeseler and H. O. Peitgen, "Newton's method and complex dynamical systems," Acta Applicandae Mathematicae, vol. 13, no. 1-2, pp. 3-58, 1988.

[23] S. Smale, "A convergent process of price adjustment and global Newton methods," Journal of Mathematical Economics, vol. 3, no. 2, pp. 107-120, 1976.

[24] E. Kreyszig, Introductory Functional Analysis with Applications, John Wiley \& Sons, 1978.

[25] W. C. Brown, Matrices Over Commutative Rings, Marcel Dekker, New York, NY, USA, 1993.

[26] E. R. Lorch, "The theory of analytic functions in normed Abelian vector rings," Transactions of the American Mathematical Society, vol. 54, pp. 414-425, 1943.

[27] J. A. Ward, "From generalized Cauchy-Riemann equations to linear algebras," Proceedings of the American Mathematical Society, vol. 4, no. 3, pp. 456-461, 1953.

[28] R. D. Wagner, "The generalized Laplace equations in a function theory for commutative algebras," Duke Mathematical Journal, vol. 15, pp. 455-461, 1948.

[29] P. W. Ketchum, "Analytic functions of hypercomplex variables," Transactions of the American Mathematical Society, vol. 30, no. 4, pp. 641-667, 1928.

[30] R. S. Pierce, Associative Algebras, Springer, Berlin, Germany, 1982. 
[31] L. V. Ahlfors, Complex Analysis, An Introduction to the Theory of Analytic Functions of One Complex Variable, McGraw-Hill, New York, NY, USA, 3rd edition, 1979.

[32] R. E. Greene and S. G. Krantz, Function Theory of One Complex Variable, vol. 40 of Graduate Studies in Mathematics, American Mathematical Society, Providence, RI, USA, 2nd edition, 2002.

[33] S. G. Krantz, Function Theory of Several Complex Variables, 1992. 


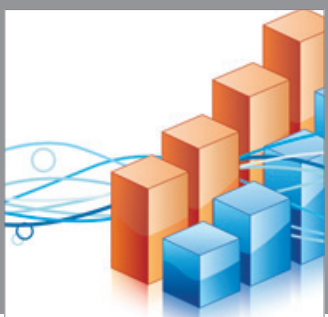

Advances in

Operations Research

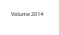

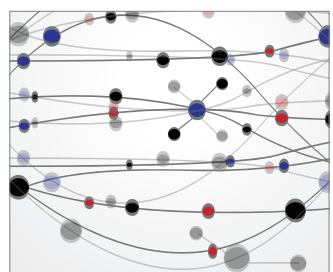

\section{The Scientific} World Journal
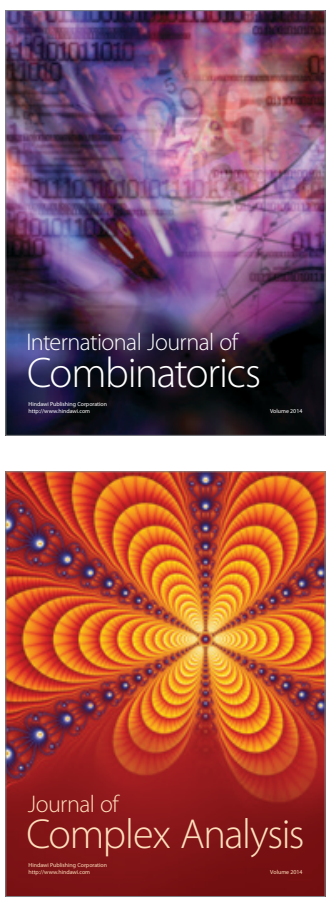

International Journal of

Mathematics and

Mathematical

Sciences
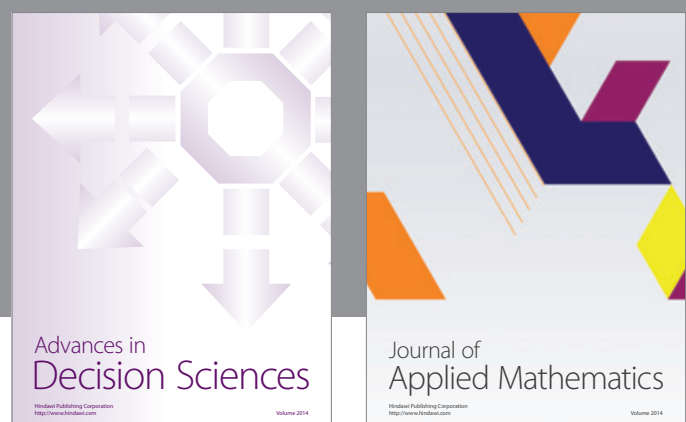

Journal of

Applied Mathematics
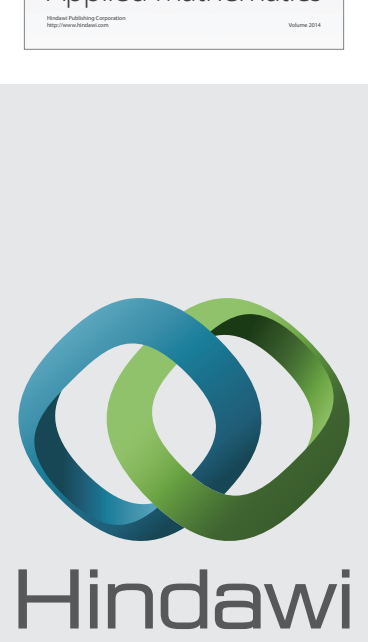

Submit your manuscripts at http://www.hindawi.com
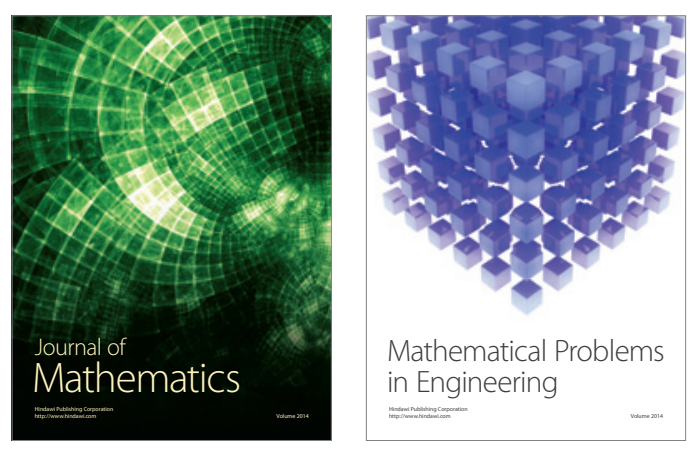

Mathematical Problems in Engineering
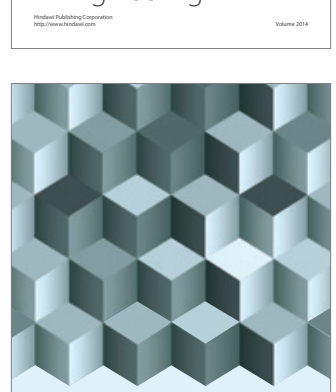

Journal of

Function Spaces
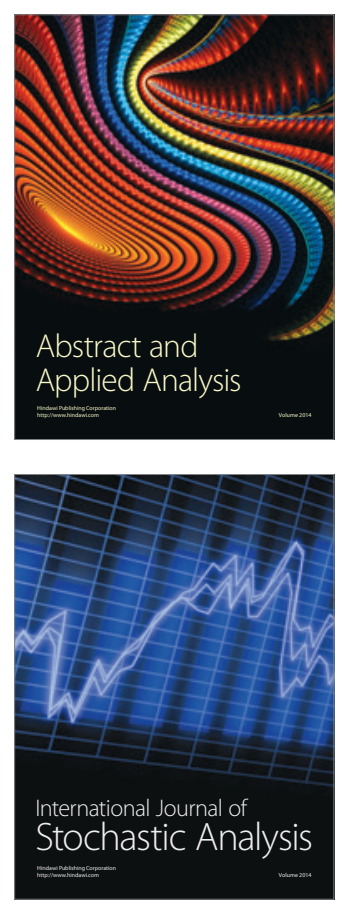

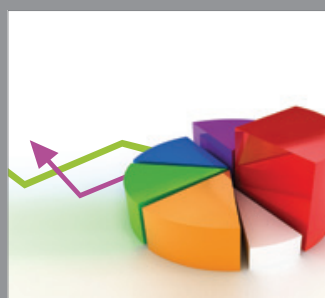

ournal of

Probability and Statistics

Promensencen
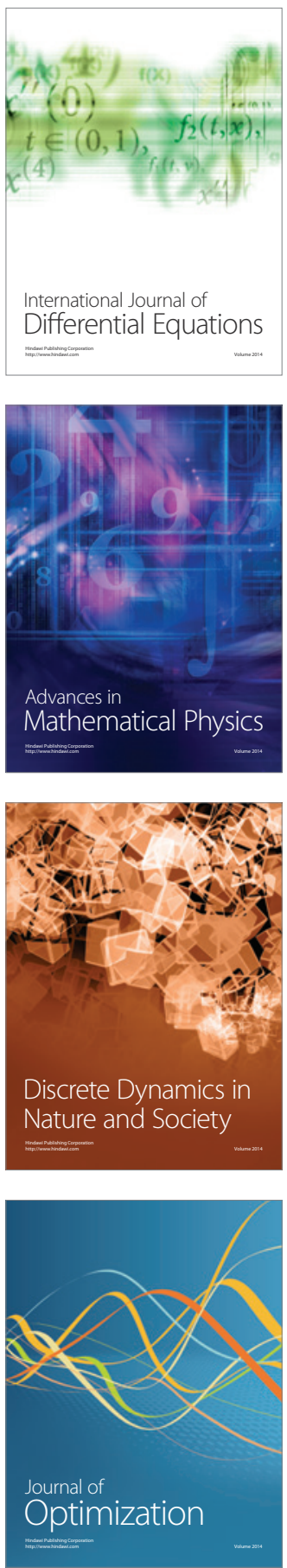